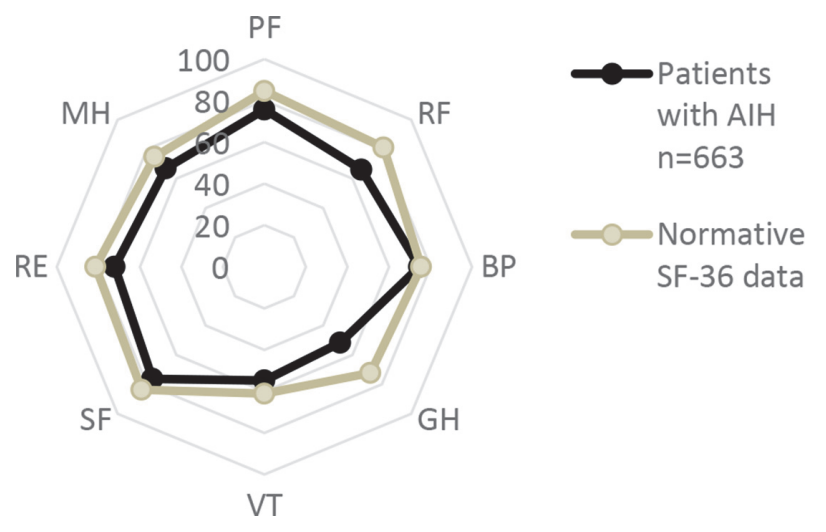

Abstract P182 Figure 1 Pooled mean SF-36 scores in patients with AlH vs. controls

Mean SF-36 subscale scores were available in 6 studies (633 patients). Pooled analysis indicated greater patient-control differences in the physical subscales GH and RF (figure 1). PCS scores were reduced more than MCS scores in four studies.

In a case-control study, 11\% $(\mathrm{n}=103)$ patients with $\mathrm{AIH}$ had PHQ-9 scores indicating clinically relevant depression (PHQ-9>10), significantly more than controls $(\mathrm{p}<0.001)$. Severe anxiety (GAD-7>15) was more common in AIH vs. controls in this study $(p=0.006)$ and STAI scores were higher (STAI1 $\mathrm{p}<0.001$, STAI2 $\mathrm{p}=0.04)$ in another.

Four studies compared disease activity and QoL, one of which found an association. Seven studies looked at association of cirrhosis with QoL, three found impaired physical (not mental) health, with no association in four. In two studies reporting comorbidities, these correlated with worse SF-36 physical subscales (RP, BP, GH). There were associations (one study each) between corticosteroid use and lower EQ-5D-5L Utility Index, with depression (PHQ-9), and with worry scores (CLDQ).

Conclusions Patients with AIH have impaired QoL compared to the general population, in both physical and emotional components. Studies to date are heterogenous and it is difficult to compare results. Further research is needed to fully explore the impact of AIH on QoL.

\section{P183 SIGNIFICANT TREATMENT SIDE EFFECT BURDEN IN AUTOIMMUNE HEPATITIS EMPHASISES NEED FOR NEW THERAPIES}

${ }^{1}$ Jessica Leighton* ${ }^{2}$ Charlotte Lloyd, ${ }^{3}$ Lin Lee Wong, ${ }^{4}$ Ann Brownlee, ${ }^{4}$ Penney Gray, ${ }^{5}$ Emma Culver, ${ }^{6,7}$ Neil Halliday, ${ }^{8}$ Michael Heneghan, ${ }^{1,9}$ David Jones, ${ }^{1,9}$ Jessica Dyson. ${ }^{1}$ Newcastle Upon Tyne Hospitals Nhs Foundation Trust, Newcastle Upon Tyne, UK; ${ }^{2}$ Newcastle University Medical School, Newcastle Upon Tyne, UK; ${ }^{3}$ Royal Devon and Exeter Hospital, Exeter, UK; ${ }^{4}$ AlH Support, UK; ${ }^{5}$ John Raddliffe Hospital, Oxford, UK; ${ }^{6}$ University College London, London, UK; ${ }^{7}$ Royal Free Hospital, London, UK; ${ }^{8}$ King's College Hospital, London, UK; ${ }^{9}$ Newcastle Biomedical Research Centre, Newcastle Upon Tyne, UK

\subsection{6/gutjnl-2020-bsgcampus.258}

Introduction Data from the national cohort study for autoimmune hepatitis (UK-AIH) demonstrates reduced health-related quality of life (QOL) for patients with $\mathrm{AIH}$, related to ongoing disease activity and, in particular, corticosteroid treatment ${ }^{1}$. To explore those findings, this International AIH Survey on Patients' Views and Experiences was designed to collect information relating to current and future treatments for AIH.

Methods The survey was co-designed by clinicians caring for patients with $\mathrm{AIH}$ and patient representatives. It was distributed electronically and the weblink disseminated to patients by AIH Support, LiverNorth and the British Liver Society, and available to patients in any country. Data was collected anonymously and descriptive analyses were undertaken.

Results There were 270 survey respondents, including 13 posttransplant patients. The median age was 55 [range 17-83 years] and 94\% were female. Age at diagnosis was 48 (1-77) years with duration since diagnosis 2.5 (0-67) years. 157 (58\%) patients self-reported having another immune-mediated disease and $49(18 \%)$ an overlap syndrome. Overall, 172 (64\%) felt they had experienced side effects (SE) from their AIH treatment. Only 107 (42\%) recall potential SE being discussed prior to starting treatment. Table 1 summarises the commonest treatments and the associated patient-reported SE. $109 / 175(62 \%)$ said they would definitely or probably take part in a clinical trial to look for better drugs for AIH.

\begin{tabular}{lllll} 
Abstract P183 & Table 1 & & \\
\hline & Prednisolone & Azathioprine & Budesonide & $\begin{array}{l}\text { Mycophenolate } \\
\text { mofetil }\end{array}$ \\
\hline Currently taking & $110(41)$ & $150(56)$ & $40(15)$ & $42(16)$ \\
Ever taken & $199(74)$ & $203(75)$ & $71(26)$ & $49(18)$ \\
Experienced SE & $112(56)$ & $73(36)$ & $19(27)$ & $9(18)$ \\
Commonest SE & $37 \%$ cognitive & $32 \%$ & $54 \%$ cosmetic & $23 \%$ cosmetic \\
(proportion of total & or fatigue & gastrointestinal & $17 \%$ & $15 \%$ \\
reported for & $35 \%$ cosmetic & $21 \%$ cosmetic & cognitive & gastrointestinal \\
medication) & & & & \\
\hline
\end{tabular}

Conclusions This survey demonstrates that patients perceive treatment side effects to be a significant problem in AIH. Over half the cohort experienced SE with prednisolone, most commonly attributing cognitive symptoms or fatigue to this medication. The impact of SE is demonstrated by patients' willingness to be involved in trials of novel therapeutics. This should encourage the community to develop further clinical trials and prioritise quality of life for $\mathrm{AIH}$ patients.

\section{REFERENCE}

1. Wong LL, et al. 2018:68(4):1487-97.

\section{P184 THE NEED FOR A MORE HOLISTIC APPROACH TO MANAGING PATIENTS WITH AUTOIMMUNE HEPATITIS}

${ }^{1}$ Charlotte Lloyd*, ${ }^{2}$ Jessica Leighton, ${ }^{3}$ Lin Lee Wong, ${ }^{4}$ Ann Brownlee, ${ }^{4}$ Penney Gray, ${ }^{5}$ Emma Culver, ${ }^{6,7}$ Neil Halliday, ${ }^{8}$ Michael Heneghan, ${ }^{2,9}$ David Jones, ${ }^{2,9}$ Jessica Dyson. ${ }^{1}$ Newcastle University Medical School, Newcastle Upon Tyne, UK; ${ }^{2}$ Newcastle upon Tyne Hospitals NHS Foundation Trust, Newcastle Upon Tyne, UK; ${ }^{3}$ Royal Devon and Exeter Hospital, Exeter, UK; ${ }^{4}$ AIH Support, UK; ${ }^{5}$ John Radcliffe Hospital, Oxford, UK; ${ }^{6}$ University College London, London, UK; ${ }^{7}$ Royal Free Hospital, London, UK; ${ }^{8}$ King's College Hospital, London, UK; ${ }^{9}$ Newcastle Biomedical Research Centre, Newcastle Upon Tyne, UK

\subsection{6/gutjnl-2020-bsgcampus.259}

Introduction Patients with chronic disease often have complex medical and psychosocial needs. Data shows that patients with autoimmune hepatitis (AIH) have impaired quality of life. This 